\title{
BIOMARKERS TO ASSESS THE ANTIOXIDANT RESPONSE IN FISH UNDER STRESS DURING THE SALMON PRODUCTION CYCLE
}

\author{
Aarón Oyarzún 1,2, Yazmín Fuentes 1,2, Daniela Escrig 1,2, Deborah Vargas 1,2, Andrea \\ Garcés 1, Sebastián Reyes-Cerpa 1,2, Geraldine Mlynarz 1, Mónica Imarai 1,3, Ana María \\ Sandino 1,2§
}

1 Ictio Biotechnologies, Alameda 3363, Estación Central, Santiago Chile.

2 Laboratorio de Virología, Centro de Biotecnología Acuícola, Facultad de Química y Biología, Universidad de Santiago de Chile, Alameda 3363, Estación Central, Santiago, Chile.

3 Laboratorio de Inmunología, Centro de Biotecnología Acuícola, Facultad de Química y Biología, Universidad de Santiago de Chile, Alameda 3363, Estación Central, Santiago, Chile.

KEYWORDS: Oxidative Stress, biomarkers, salmonids, aquaculture, fisheries.

§ Corresponding author. Tel.: +56 227181114. E-mail address: ana.sandino@usach.cl

Oxidative stress generates high levels of reactive oxygen species (ROS), resulting in damage to lipids, proteins and DNA, changes in glutathione and antioxidant enzymes. Test oxidative stress in fish during farming can be easily done by quantify membrane damage by lipid peroxidation, whilst the antioxidant defense can also be evaluated by measuring the ratio of oxidized and reduced glutathione. Instead, quantity changes in antioxidant enzymes are quite cumbersome, as it will require knowing more about kinetics and type of responses. With this study, we seek to obtain biomarkers to follow up oxidative stress in fish during farming. Lipid peroxidation and glutathione was measured by colorimetric methods to evaluate the antioxidant response of fish under stress due to various farming practices. 30 to 60 fish from each batch of farmed fish, both in open and in recirculation farming, at times considered the most critical in the process of freshwater, were sampled as indicated: 7-10 days before and after selection or grading, 7-10 days before and after vaccination, 7-10 days prior to transfer to seawater (pre-smolt stage) and 7-10 days after (smolt stage). The results showed that lipid peroxidation consistently increased with each one of the practices that was concomitant with an increase of the reduced glutathione, apparently as a way of defense of fish. Interestingly, fish in circulating aquaculture farm were more stressed than those of an open aquaculture farm. Expression of cytokines like IL-10, TGF- $\beta 1$, IFN-I y II, and of C3, CD4 genes of the same fish are currently under quantification to see the potential impact of stress on immune response. Altogether, results showed that to assess levels of lipid peroxidation and glutathione in fish are good biomarkers of stress produced during farming. Hence to counteract this oxidative activity one can be able to introduce some changes in farming practices and/or use of supplements or additives in the diets, thus improving the physiological condition of the fish. 